\title{
Neuroendoscopy Adapter Module Development for Better Brain Tumor Image Visualization
}

\author{
Sunil L. Bangare ${ }^{1}$, G. Pradeepini ${ }^{2}$, Shrishailappa T. Patil ${ }^{3}$ \\ ${ }^{1,2}$ Department of Computer Science \& Engineering, K.L. University, Guntur, A.P. India \\ ${ }^{3}$ Department of Computer Engineering, V.I.T., Pune, India
}

\begin{tabular}{l}
\hline \hline Article Info \\
\hline Article history: \\
Received Jan 13, 2017 \\
Revised Aug 11, 2017 \\
Accepted Aug 30, 2017 \\
\hline
\end{tabular}

Keyword:

Neuroendoscopy adapter module (NAM)

Support vetor machine (SVM)

Particle swarm optimization

(PSO)

Subdural haematoma

Medical imaging

Classification

Visualization

\begin{abstract}
The issue of brain magnetic resonance image exploration together with classification receives a significant awareness in recent years. Indeed, various computer-aided-diagnosis solutions were suggested to support radiologist in decision-making. In this circumstance, adequate image classification is extremely required as it is the most common critical brain tumors which often develop from subdural hematoma cells, which might be common type in adults. In healthcare milieu, brain MRIs are intended for identification of tumor. In this regard, various computerized diagnosis systems were suggested to help medical professionals in clinical decision-making. As per recent problems, Neuroendoscopy is the gold standard intended for discovering brain tumors; nevertheless, typical Neuroendoscopy can certainly overlook ripped growths. Neuroendoscopy is a minimally-invasive surgical procedure in which the neurosurgeon removes the tumor through small holes in the skull or through the mouth or nose. Neuroendoscopy enables neurosurgeons to access areas of the brain that cannot be reached with traditional surgery to remove the tumor without cutting or harming other parts of the skull. We focused on finding out whether or not visual images of tumor ripped lesions ended up being much better by auto fluorescence image resolution as well as narrow-band image resolution graphic evaluation jointly with the latest neuroendoscopy technique. Also, within the last several years, pathology labs began to proceed in the direction of an entirely digital workflow, using the electronic slides currently being the key element of this technique. Besides lots of benefits regarding storage as well as exploring capabilities with the image information, among the benefits of electronic slides is that they can help the application of image analysis approaches which seek to develop quantitative attributes to assist pathologists in their work. However, systems also have some difficulties in execution and handling. Hence, such conventional method needs automation. We developed and employed to look for the targeted importance along with uncovering the best-focused graphic position by way of aliasing search method incorporated with new Neuroendoscopy Adapter Module (NAM) technique.
\end{abstract}

Copyright @ 2017Institute of Advanced Engineering and Science. All rights reserved.

Corresponding Author:

Sunil L. Bangare,

Research Scholar, Department of Computer Science \& Engineering,

K. L. University, Guntur, Andhra Pradesh, 522 502, India

Email: sunil.bangare@gmail.com 


\section{INTRODUCTION}

The aim of this analyze is to provide and compare three perfectly computerized systems for rapid classification of brain MR images into healthy, and brain with the tumor infection. It functions in of four actions:

(i) Segmentation of the image to get image geometric boundaries of tumor cells;

(ii) Computation of the AFD signature of the image;

(iii) Extraction of the features of the provided AFD; and

(iv) Classification by Neuroendoscopy Adapter Module (NAM) along with performance comparison against support vector machine (SVM).

The PSO algorithm [1] is employed for pre-processing the MR image to split up the vital areas of the brain and additionally boost the detection of those which indicates high-intensity contrast as regarding tumor. The purpose is to identify tumor by discovering the pixel level dissimilarities around normal and abnormal tissue as well as the presence of active boundary edges. With this circumstance, the MRI pixel set is an issue, and to identify a pixel partitioning that enhances the between-class deviation of the distribution of intensity degrees. As per traditional solution, PSO hinges on an etiological metaphor to resolve these kinds of issues with stochastic search global optimization. It is one of the so-called evolutionary computation paradigms in computational intelligence. Primarily PSO was accustomed to achieving the brain MRI segmentation task due to the performance to produce approximate ways too complicated optimization issue with the competitive computational cost [2]. Furthermore, PSO is more rapidly and consistent than genetic algorithms and ant colony optimization [3] but, in real life application, since the complexity and nonlinearity of many problems, the target functions of these problems are often discrete and of multi-point value, furthermore, the modeling the problem itself is also very difficult [4]. In this work, we compared PSO, SVM, and newly developed NAM performances. NAM enables discovering the existence of high-intensity bursts in the array of the image and consequently using them as image signatures to distinguish the common form of tumor affected MRIs.

Many of the previous workouts report high classification accuracies, but each kind has possible negative aspects. The demand for manually-determined or established regions of interests in the MRI [5] is widely studied. The most problematic studies are overcoming the lack of boundaries, poor contrast, noise, supervised method, less robust, less efficient and less reliable to make a full use of obtaining data. Thus, it is expected that image processing methods will help to solve those problems [6]. Also, the chance of corrupting the feature set using noise along with artifacts due to applying extensive echo MRS [7] and also conducting categorization instead of supervised classification [8], categorization being less suitable for clinical diagnosis. Eventually, even though solution in [9] brought about an overall gain in computation time without any decline in accuracy, it is not worthy of massive multi-datasets [10]. Furthermore, the offered model in [11] is dependent on quite a few constraints to be tuned and a multitude of features, as well as being susceptible to confusing tumor boundaries. Lastly, the effort in [12] necessitates the proper detection of a region of interest conducted manually.

Hence, as of the fact auto-focus algorithm have to be real-time, the conventional auto-focus algorithm could have numerous difficulties as a consequence of increasing computations as the volume of windowpane area location can vary considerably usually. A particular problem stands out as the repeated interpolation of auto-focus receives retarded as a consequence of increasing calculations. Additionally, the likelihood linked to de-focusing of targeted image could surge because of the mismatch with the algorithm performance and also a result of the target. The local-aliasing-sampling method is evidently applied to lessen the computation, especially in medical-microscopic-dig-cam. However, inside the Local-aliasing technique, many accurate specifics may lose in conjunction with the increase in noise component [13]. As a result, the lens deviates throughout the exact position along with picture blur may perhaps take place. To defeat this concern, the proposed approach "NAM interpolation" evaluates every single misplaced pixel through an accumulation of texture-relevant semi-local pixels when using the regularity similarity recurrently computed through some sections of several proportions. Notably, implementing NAM-iteration, crucial clogging texture pixels are involved in producing symptoms as well as posterior analysis, and a noticeably bilateral detailed transform is essential for regularization of unnecessary clogging /plasma layer removal from the image. Trial and error effects considered next to existing interpolation techniques confirm our approach are unable just considerably to slow up the aliasing difficulty but also acquire significantly better outcomes throughout a range of views just as concerning quantitative evaluation together with very subjective image quality. The introduced algorithm may be additionally utilized as a possible API for digicam image quality improvement. To summarize, employing an entirely new neuro-endoscopy technique jointly with autofocus imaging increased field of vision of tumor ripped growths, corresponding to the presence attained using chromo neuro-endoscopy. 
Sehgal, Aastha, et al. (2016) proposed method for the image diagnosis process for brain cancer, clinicians often need to achieve a brain biopsy to get histological data. However, there are risks associated with this procedure: 1) collected samples are not always representative of the tumor and 2) there is a risk of blood vessel rupture when the sample is taken. This type of bleeding occurs between 0.3 and $59.8 \%$ of the cases, and the mortality rate can be as high as $3.9 \%$. Here author presented a diffuse reflectance spectroscopy imaging system directly integrated on a brain biopsy needle to guide the surgeon during needle biopsy procedures [14]. But this technique lacks in pre or post-surgical analysis.

Also, as per Mohyeldin, Ahmed, et al. (2016), the object of their study was to assess the feasibility, accuracy, and safety of real-time MRI-compatible frameless stereotactic brain biopsy. The ability to place a biopsy cannula under real-time imaging guidance permits on-the-fly alterations in the cannula trajectory and tip placement. Real-time imaging during MRI-guided brain biopsy provides precise, safe targeting of brain lesions. But, again this technique lacks in image enhancement and usability [15].

Magnetic resonance imaging underestimates histologically identified tumor boundaries, particularly for wounds that have a high imaging suspicions score and a high Gleason score [16]. A $9 \mathrm{~mm}$ treatment perimeter all around a lesion visible on magnetic resonance imaging would certainly consistently make positive treatment method of the entire histological tumor volume during focal ablative therapy. However, the clogging layer is present with tumor cancer growth hence it is necessary to remove aliasing effect arising due to clogging /plasma infusion. We are focusing on brain cancer case, but the solution by NAM may be for all types of region tumor detection.

The earlier investigation has mentioned that Neuroendoscopy resection for tumor growths ends up with a 76-90\% decrease [17] in the chance of acquiring tumor melanoma. Even so, elements like endoscopist's insertion along with revulsion ability, cancerous growth position, and quality of colon prep, and cancerous growth depiction can lessen the potency of Neuroendoscopy tests. For example, 3 to 4 Ripped tumors are typically overlooked through Neuroendoscopy, as they are complicated to discover when using the typical white-light image resolution technique.

Despite the fact that, neuro-endoscopy gives benefits more than traditional Neuroendoscopy, the method is more complicated as well as time-consuming. Nowadays, image-enhanced neuro-endoscopy continues to be recommended to further improve microvascular comparison along with the assist in the instant image resolution of simple patterns and also shade variances. A digital graphic improvement entails image processing algorithms. Different algorithms are already recommended according to the targets and also the modality of image resolution, which includes the one which improves the shape or light patterns within the produced graphics, one particular focusing image comparison, one intended for color transformation and so forth. The application of existing strategies like auto resolution and also small image resolution can result in enhancements in tumor growth diagnosis rates, despite the fact that this idea is debatable [18]. Also, Johns Hopkins neurosurgeon may be using fiducial markers to target certain areas for surgical resection or treatment. Before the surgery, a CT or MRI scan with the fiducials in place creates a 3-D map of your head. During operation, the fiducially provide a series of reference points for the surgeon and integrate navigation during surgery with the 3-D map (http://www.hopkinsmedicine.org/). This imaging-assisted technique helps doctor safely find the way around the brain and precisely locate tumors, targets for deep brain stimulation and other lesions in three dimensions as he or she works, almost like a GPS system. This kind of combined outcomes has been acquired when using the neuro-endoscopy technique one generation before the present one.

We collected existing results of the retrospective analysis for evaluation of visibility of tumor tested with PSO as well as SVM graphics, but we did not test 3D imaging technique. Exclusively, we focused on ascertaining whether or not the analysis abilities of SVM and PSO were better than that of our newly developed NAM (Neuroendoscopy Adapter Module) for the visualization of tumor growths which often surrounded by uneven clogging layer. Many times, such clogging layers create visibility obstruction for doctors. Hence, many times to predict the cancerous growth at initial investigation become ambiguous.

\section{RESEARCH METHOD}

Neuroendoscopy graphics obtained from MultiSpeciality Hospital patient (identity/privacy protected) at the Pune, India had been used for this current research. Existing results were obtained from web/physician's data that carried out NAM and UQI by transitioning initial towards SVM method accompanied by the PSO manner. Doctors used the following equipment in this study: CT, MRI imaging, light sources, and video processors.

Ultimately, the SVM classifier [19] is employed to distinguish between normal and tumor brain images influenced by fractal features. The SVM is dependent on the statistical learning principle which locates the suitable nonlinear hyper plane that decreases the anticipated classification error. It was 
subsequently efficiently implemented in previous works [20-22]. SVM proved to be most efficient in iris image processing, feature extraction by using independent component analysis. The support vector machine is used for iris classification and recognition [23]. The SVM method is particularly important in the field of machine learning. There are many advantages of the SVM, such as direct geometric interpretation, elegant mathematical tractability, and high accuracy [24]. SVM becomes famous when, using pixel maps as input; it gives accuracy comparable to sophisticated neural networks with elaborated features in recognition of task [25]. Hence, we adopted SVM advantages to build new algorithm.

We provide a new solution to detect the tumor in brain magnetic resonance images. First, the recommended automated system is easy to employ and to understand. It is mostly based on segmentation of biological tissue, evaluation of the directional spectral distribution of the segmented image, and evaluation of multi-scale fractals of the calculated directional spectral distribution. Certainly, the existence of tumor influences the homogeneity of the biological tissue and also its geometrical behaviors. In this aspect, directional spectral distribution enables characterizing geometrical patterns manifested by segmentation outcomes and as well textural features of the biological tissue. These patterns can be considered at various scales by employing fractal estimates. Second, we assess the performance of the proposed system under NAM algorithm is accustomed to focusing the tumor affected region. NAM algorithm is interesting as it helps to focus on segmentation approach with the focus on tumor edge correctness visibility. Third, unlike previous works [16-18], our proposed system doesn't need manually-determined or established regions of interests. Also, our automated detection system is fast and provides high accuracy; unlike the MRI technique which is used in the image classification process that generates real-time pseudographics. In further section 2.1, we presented computational methods used during current research analysis.

\subsection{Computational Methods}

The overall tumor/subdural hematoma detection and analysis system is described in the Figure1, where brain MRI is segmented by PSO/SVM/NAM. The obtained segmented image is further processed to obtain its Auto-focus Distribution (AFD) which automatically cuts down peripheral focus and improves the pixel boundary visibility of tumor. Indeed, AFD is suitable to characterize texture of brain biological tissue. Then, UQI is employed to compute the pixel quality of the AFD of input brain images. In this regard, those fractals are used to form the features vector. Finally, the NAM is trained with the features vector to classify a given brain MRI/neuro-endoscopy image as a pre-surgical or post-surgical image to identify whether or not tumor affected portion is clear. All techniques; including PSO-based segmentation algorithms, SVM and NAM, are described in next subsections.

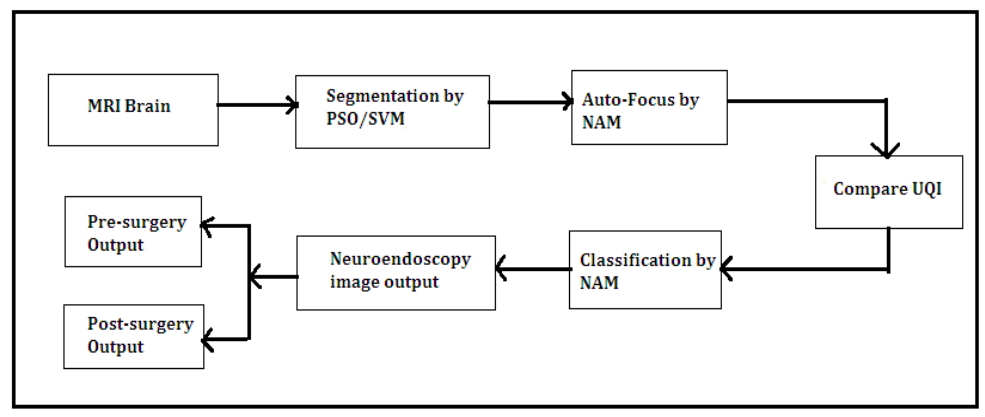

Figure 1. Proposed system for tumor detection and surgical analysis

\subsubsection{Particle Swarm Optimization Techniques}

Particle swarm optimization (PSO) is a population-based search protocol presented by Salim Lahmir [26]. In the image segmentation framework, the pixel set matches to the lookup space, along with the optimum solution matches to exploiting the between-class deviation of the distribution of intensity degrees in the image. Following the notation in [27] notation, at time $t$ each particle $n$ moves in a search space with position $X_{t}^{n}$ and velocity $V_{t}^{n}$ which are dependent on local best position $P_{t}^{n}$ neighborhood best $N_{t}^{n}$ and global best $G_{t}^{n}$ information as follows:

$$
v_{t+1}^{\eta}=w v_{t}^{\eta}+\rho_{1} r_{1}\left(\breve{g}_{t}^{\eta}-x_{t}^{\eta}\right)+\rho_{2} r_{2}\left(\breve{x}_{t}^{\eta}-x_{t}^{\eta}\right)+\rho_{3} r_{3}\left(\breve{n}_{t}^{\eta}-x_{t}^{\eta}\right)
$$


$x_{t+1}^{\eta}=x_{t}^{\eta}+v_{t+1}^{\eta}$

Where the parameters $w, p_{1}, p_{2}$, and $p_{3}$ are respectively the weights of the inertial influence, global best, local best, and neighborhood best when determining the new velocity. The parameters $r_{1}, r_{2}$, and $r_{3}$ are vectors with uniform random numbers between 0 and 1 associated to each component of Eq. (2). The fitness function $\phi$ used to evaluate the performance of the particles is given by [27][28]:

$$
\begin{aligned}
& \max \sigma\left(t_{j}\right) \\
& \Phi=1<t_{1}<\cdots<t_{\eta-1}<L
\end{aligned}
$$

where, $\sigma$ is the between-class variance of the image intensity distributions, $t$ is a given threshold, $L$ is the intensity level, and $n$ is the number of classes into which the pixels ofthe image are divided (by grouping pixels in terms of their gray levels been within a specified range).

The $S C_{c}^{\max }$ is given by [29] to solve the problem with PSO algorithm that it may get stuck in a suboptimal solution region [30] [31]. In order to solve this problem, we introduced the NAM where pixel selection is employed to escape from local optima. In this context, when a maximum threshold number of steps $S C_{c}^{\max }$ is reached without improving the fitness function, the worst particle is deleted. The steps counter $S C_{c}^{\max }$ is given as:

$$
S C_{c}\left(N_{k i l l}\right)=S C_{c}^{\max }\left(1-\frac{1}{N_{k i l l}+1}\right)
$$

where $N_{\text {kill }}$ is the number of particles deleted from the swarm when there is no improvement in fitness during an iteration. A new swarm is created with a probability of $p=f / S$, with $f$ is a random number in $[0,1]$ and $S$ is the number of swarms. Artificial Intelligence techniques related to brain are discussed by Sutikno Tole et al [32].

Fractional calculus based on the concept of fractional differential is used to control the convergence rate. The fractional differential of a given signal $x(t)$ is given by:

$$
D^{\alpha}[x(t)]=\frac{1}{T^{\alpha}} \sum_{k=0}^{t} \frac{(-1)^{k} \Gamma(\alpha+1) x(t-k t)}{\Gamma(k+1) \Gamma(\alpha-k+1)}
$$

Where $\square, T$, and $r$ are respectively the gamma function,the sampling period, and the truncation order.

\subsubsection{Support Vector Machine Classifier}

Given a training set $\{(\mathrm{xi}, \mathrm{yi}), \mathrm{i}=1,2, \ldots, \mathrm{m}\}$, where the input $X_{i} \in R_{d}$, and class labels $Y_{i} \in$ $\{+1,-1\}$, the separation hyper-plane for a nonlinear SVM classifier [22] has the following form:

$$
g(x)=\operatorname{sign}\left(\sum_{i=t}^{m} y_{i} \alpha_{i} K\left(x x_{i}\right)+b\right)
$$

where $K$ is a kernel function (for instance, a polynomial kernel), is the Lagrange multiplier, and $b$ is a parameter to be estimated. In this work, the polynomial degree was varied from 2 to 4 . Higher orders were ignored because of a higher computational burden with no substantial gain in classification accuracy from our experiences. The leave-one-out cross-validation method is adopted for validation of experiments, and average and standard deviation of accuracy rate, sensitivity and specificity are calculated to evaluate the performance of the SVM. In our work, positive samples and negative samples are respectively pre-surgical tumor and post surgical images. The NAM is adopted as main classifier although different ones may be considered and compared using UQI. Further section 2.1.3 provides the NAM technique. Few datamining algorithms proposed by Abdar, Moloud, et al. were also studied [33]. S. L. Bangare et al. have proposed the methods of Brain tumor identification, classification and SVM, which were also studied for this experiment [34] [35] [36].

\subsubsection{Neuro-Endoscopy Adapter Module}

The NAM technique enables instant modality transitioning through PSO and SVM employing a Matlab control interface. We can easily use real time images captured by MRI and neuro-endoscopy without having magnification had been taken and digitally aged in electronic digital health care records. The graphics were being determined by a highly skilled physician blinded to the present study's approaches. The NAM, 
SVM and PSO graphics for every single lesion had been delivered electronically, arbitrarily organized, and a Microsoft PowerPoint demonstration was developed. These kinds of images produced by NAM consist of fine details which could distinguish the individual or the lesion attributes for normal brain, pre-surgical and post surgical brain images. Skilled health professionals took part in the image assessment by visual analysis and compared technically using UQI.

The MRI and Neuroendoscopy graphics have been introduced to each one of the doctors in arbitrary order for comparability using the NAM. Doctors obtained each one of the CT, MRI and neuro-endoscopy graphics for visibility of ripped tumors based on the pursuing range: 0 (good), +2 (better),+3 (excellent in case of NAM), -2 (poor), and also -1 (very poor). The feasible highest ranking for almost any graphic had been +7 , along with the probable lowest ranking had been -7 . If a perception acquired a complete ranking of +7 or even more, the graphic was thought to be enhanced, a ranking in between +8 and -8 suggested no transform, along with a ranking of -10 or a smaller amount pointed out diminished presence. This work moreover analyzed the existing Neuroendoscopic image (refer Figure 2 bellow) for further comparison with NAM output images (refer Figure 3 in Section 3). Dissimilarities between images have been analyzed by visual, UQI and SPSS test. Hence, using Yamen's formula we got P-value <0. 05 which means NAM method is superior than SVM and PSO.

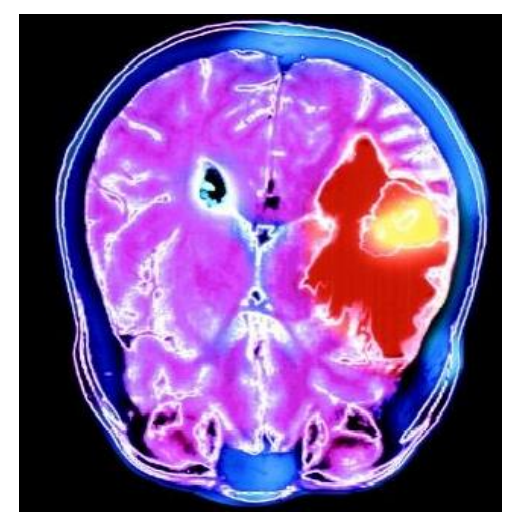

Figure 2. Original Neuroendoscopy (Subdural Hematoma) image (Source: Patient Test Report, 2016)

\section{NAM ITERATIVE INTERPOLATION: CLINICAL CASE STUDY}

Despite the advantages, there are some complicating issues around neuroendoscopy that might not always be easily tackled or avoided. Performing a neuroendoscopic procedure for Subdural Hematoma requires a highly specialized and trained neurosurgeon, meaning the availability of this procedure is normally restricted to a small number of specialized medical facilities [28].

The operative environment for Subdural Hematoma is understandably bloody, which might compromise the surgeon's field of vision, thus demanding a regular cleansing of the endoscope. Blood clogging is also a possible problem that requires the endoscope to be removed to be properly cleaned, and then placed back again inside the patient's brain. Finally, the operating Subdural Hematoma sheath's small diameter limits the working channels and this, in turn, hampers the instruments' movements. Also, voronoi brain mapping gives similar disadvantage for real time brain tumor detection and image visualization. Hence, focusing over to overcome such disadvantages we developed the NAM technique which can alias the clogging /blood effect and neurosurgeons can able to view clear state of brain tumor.

In line with the opinion through which standard image characteristics repeated similar locations combined with the assessment of anti-aliasing in the earlier segment, we provide a continuing Neuroendoscopy auto-focus interpolation strategy of anti-aliasing along with locating the high-quality image. In this article, we will take in the NAM interpolation of purely an individual iteration in addition to particular location measurement. Inside the following, we will certainly describe easy methods to assess region similarity, associated with the area analysis for dropped pixel importance calculation.

Regular deviation refines the result involving window in addition to sub-window focus length with ultimate covering region as shown in Figure-3. For any solitary missing pixel, function as the tolerance value to tell apart the texture-relevant pixels by means of all of the close pixels. Generally, before you start, interpolated beliefs regarding dropped pixels are usually flawed all of which deliver the dysfunction to the computation of the window dimensions. From the curiosity regarding examination, we presume the particular 
variation relating to begin with interpolated value connected with sub-window in addition to the main window-value to watch any perfection connected with image capture focus with zero as well as standard deviation.

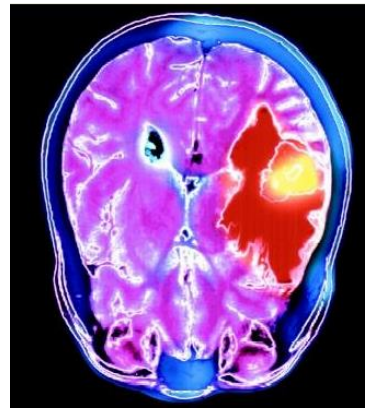

(a) Original Image

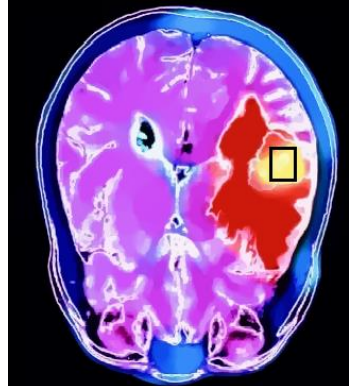

(b) NAM applied Auto-Focus Image

Figure 3. Output Images representation of Subdural Hematoma by Neuroendoscopy Adapter Module (NAM)

At this point, we can easily notice that window region $W \biguplus \mathrm{a}\left(y_{i}\right)$ is used since common region, beyond just the early interpolation untiln $\left(y_{n}\right)$. All interpolated semi-local Window areas $W \biguplus \mathrm{a}\left(x_{i}\right)$ and subwindow area $W \cup \mathrm{n}\left(y_{n}\right)$ are usually well balanced using the regular region. As explained after using this multi-scale approach, to feature the benefits of each and every significant as well as small-scale pixel location, we got positive results caused by many preceding computations (as depicted in NAM algorithm) jointly as strategies for the scaled-down measurement variation. The actual interpolated values associated with dropped pixels one of many inputs is broad as well as diverse. Window region includes merely pixels and it is continuous; as a result, the sub-window region can be used as the regular area to equal just about all close by original window places it doesn't matter what input they are through.

\section{NEUROENDOSCOPY ADAPTER MODULE (NAM)}

With the anti-aliasing perspective, it's appropriate to select larger region measurements so that they can uncover a lot more trustworthy pixels. The more important the region sizing, more close by pixels are utilized since the method to locate clogging -layer-relevant pixels as well as reduce the precise consequence related to aliasing artifacts. Nevertheless, the great textures in the conventional image require scaled-down region measurements to obtain much better quick compressed facts. The objects may appear due to region mismatching. Nonetheless, it may be difficult to consider the proper area is sizing observed image getting aliasing. With this particular document, considering both of those different items, we suggest real recurring multi-scale interpolation solutions which replicate the actual semi-local interpolation through big to scaleddown region sizing recurrently.

Algorithm 1: Neuroendoscopy Adapter Module

Input: MRI/Neuroendoscopy medical image

Step-1. Initializing boundaries of window:

(a) Initial area of image point $F_{a}=0$, Initial coordinates of window

$W_{c}=(0,0)$ i. e. start point of diagonal length of image point window

(b) Set step size as per standard deviation $W \circledast \sigma_{k}$ and area $a \cap\left(y_{k}\right)$

(c) Set $\sigma_{s}$ as the referenced standard deviation of image point for $\omega \cap\left(x_{i} y_{i}\right)$

(d) Record image boundaries by matching pixel coordinates of final window image points

(e)Store values of first window image point as a reference window image point's area

Step-2. Iterative approximation of diagonal matrix:

(a) Record curve covering coordinates of parallel lines of diagonal length

(b) Deduct window image point area from curve covering extra boundary pixels

(c) Resize window image point to remaining curve

If $W c \circledast F_{a} \leqq$ curve area

go to step-1

else

Consider next curve for pixel coordination 
Step-3. Compute diagonal coordinates of image point window and locate boundary pixels.

Step-4. Remove unfitted curve pixels.

Step-5. Map next curve within diagonal pixel points within window $W \cup c x_{i}$ and also $W \cup c y_{i}$.

It should be pointed out that this outcome originating from just about all previous iterations are usually gathered collectively as inputs towards the following little dimension version, which may be different through the traditional repeating procedure. In most solitary version, presented the region sizing together with inputs which incorporated the outcomes originating from just about all previous iterations, each and every interpolated semi-local window-port region concentrated through pixel $x_{i} \ldots \ldots x_{n}$, it doesn't matter what enter they are originating from, is actually considered towards dropped pixel $y_{i} \ldots . y_{n}$ focused area.

Right after determining most Neuroendoscopy along with related pathology studies, we ruled out reviews where:

(1) There seemed to be multiple Neuroendoscopy course of action and pathology statement about the same time;

(2) A similar MRI record identified and merged with Neuroendoscopy process;

(3) The individual identifier seemed to be lost and

(4) The particular document was produced using computer software.

The application enables a clinician to build a Neuroendoscopy document along with pre-populated drop-down choices. Considering that the studies are produced in a very organized structure, we used images captured during MRI and Neuroendoscopy. Also, we incorporated reports provided by doctors. The rest of the Neuroendoscopy reviews within our SPSS analysis test had been determined and also transcribed.

Following the implementing of the Matlab application for the reports, we joined the reviews of the individual, the physician who carried out the process along with the medical center exactly where it had been conducted. For most doctors, there was the entry to merely the particular subset of the neuro-surgery department they conducted at MultiSpeciality Hospital. Most of these doctors have been in specialty practice and carried out processes at MultiSpeciality Hospitals, and private neuro-endoscopy facilities for initial patient assessments.

As we discussed the patient clinical case details in previous Section-3 and NAM algorithmic details in Section-4, next Section-5 provides details about result and analysis of algorithmic research.

\section{RESULTS AND ANALYSIS}

MR and Neuroendoscopy brain images were obtained from the MultiSpeciality Hospital and web for validation of the proposed system. The set included 15 images of normal (healthy) brains and 15 of a brain tumor. The Matlab (C) with image processing toolbox software was used for image processing and classification. Figure-4 shows an example of normal brain MRI and its segmentation results NAM.

To quantitatively evaluate the segmentation results, the performance of each NAM-based segmentation algorithm is assessed by computing the universal quality index (UQI) (http://live.ece.utexas.edu/) which is independent of the images being tested and the viewing conditions or the individual observers. The higher the index, the better is the segmentation result. For instance, the values of UQI obtained for healthy brain and PSO processed healthy brain are 0.3619 and 0.3624 .

Also as shown in Figure 4 bellow, the values of UQI obtained for SVM and NAM for segmentation of a tumor affected brain image are respectively 0.4337 and 0.4342 . Although these values are close, the NAM algorithm yields to the best result among the machine learning algorithms.
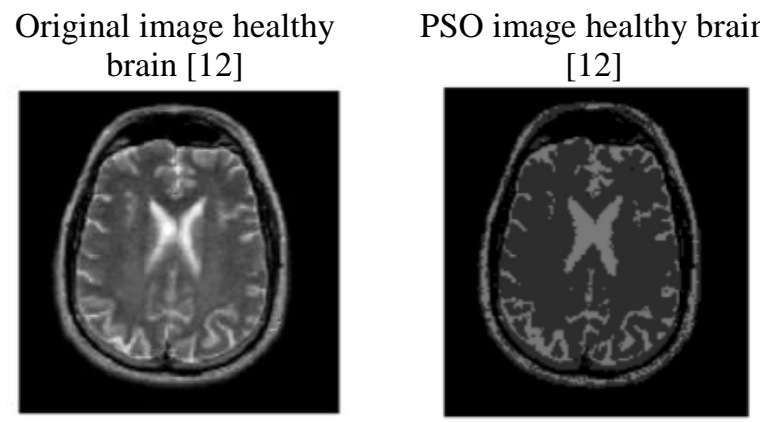

SVM tumor detection

NAM tumor detection
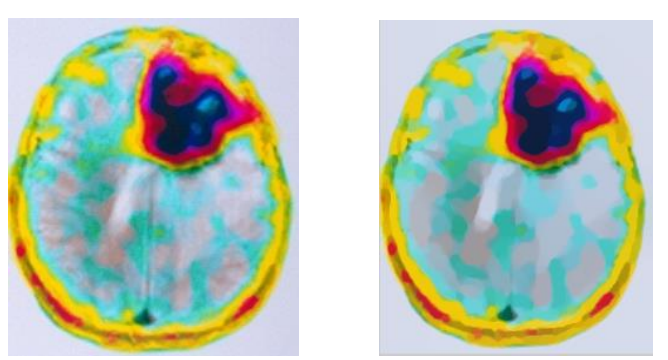

Figure 4. Segmentation results of a healthy brain (original brain image and PSO processed image) and tumor affected brain using SVM and NAM. 
Outcomes regarding NAM, SVM, and PSO are revealed in Table-1 and also NAM, enhanced visibility seemed to be identified $71.4 \%$, close by visibility appeared to be obtained in SVM 63. $1 \%$ sufficient reason for PSO, much less enhanced visibility had been seen as compared to the original image as $27.9 \%$ for the analyzed case. As shown in Table 1 bellow, the likelihood of enhanced field of vision of NAM was appreciably greater than that of SVM and PSO graphics. The NAM scores helped for being increased, in contrast to SVM and PSO scores maintained to be reduced. There seemed to be absolutely no significant difference altogether field of vision scores between histopathological conclusions. There have been no significant dissimilarities in inter-observer understanding among Neuroendoscopy graphics.

Table 1. Comparative Image Visibility Analysis

\begin{tabular}{ccll}
\hline $\begin{array}{c}\text { Medical Image } \\
\text { Technique }\end{array}$ & $\begin{array}{c}\text { Visibility (\%) as } \\
\text { per UQI }\end{array}$ & \multicolumn{1}{c}{ Doctor Remark } & \multicolumn{1}{c}{ Performance Rating } \\
\hline NAM & $71.4 \%$ & $\begin{array}{l}\text { Clear tumor region visibility with clogging } \\
\text { aliasing effect } \\
\text { Traditional performance with plasma/clogging } \\
\text { visibility around tumor } \\
\text { Comparatively less tumor region visibility }\end{array}$ & $\begin{array}{l}\text { Excellent for pre-surgical } \\
\text { investigation } \\
\text { Good for surgical events }\end{array}$ \\
SVM & $63.1 \%$ & $\begin{array}{l}\text { Good for primary tumor position } \\
\text { investigations }\end{array}$ \\
\hline
\end{tabular}

Preceding work has acknowledged this variance in quality among the disadvantages of Neuroendoscopy as being a screening process application for tumor cancer malignancy testing. Niche organizations, as well as professionals, have got requested vendors to survey on the Neuroendoscopy effectiveness, although couples of doctors are confirming their quality. Matlab could be one method of generating schedule exposure more prevalent. Our final results emphasize numerous pros and cons of employing MRI classification using PSO, SVM for the quality of measuring, applying information within image database. The significant benefit of Matlab is it is monetarily possible. Another advantage is that Matlab enables vendors to carry on making use of standard plot whenever explaining affected individual care becomes easy. The Matlab application also offers an advantage that it is usually effortlessly used to include modifications towards the quality image processing and to build deployable API interface for any medical device like neuro-endoscopy tools.

Here, we, in the beginning, carry out trials of our method combined with various kinds regularize so as to vindicate the necessity of employing the NAM approach for image enhancement. Consequently, we analyze the recommended approach with present NAM auto-focus interpolation methods on real-time medical images which are down-sampled with suggested technique in minimizing aliasing implications. Ultimately, we compared the recommended (NAM) method using existing SVM and PSO techniques to identify the performance of NAM.

In the fundamental research, we executed our NAM algorithmic approach for the Neuroendoscopy images, respectively. The experimental results are confirmed in Figure-4 which in turn displays a neighborhood location of the tumorous growth without blood clogging or plasma inclusion, which often consisting of excessive aliasing artifacts. We can quickly notice that the contour of the image pattern is restored adequately; even so, the perimeters are clear, along with the pixels be observed through the interpolated pixels. However the NAM leads to the spatial smoothness, this also additionally clears the visibility for doctors. Refer Table 2 bellow for evaluation of NAM Algorithm performance together with existing SVM/PSO technique.

Table 2. Comparison of NAM Algorithm Efficiency with SVM/PSO Method.

\begin{tabular}{ccccc}
\hline Image & \multicolumn{2}{c}{ SVM/PSO Method } & \multicolumn{2}{c}{ NAM Algorithm } \\
& Min & Diff & Min & Diff \\
\hline MRI and Neuroendoscopy Test Input & 31.43 & 0.711 & 36.32 & 0.842 \\
\hline
\end{tabular}

Eventually, the pixels are recognizable despite the fuzzy interpolated forms. Figure-4 reveals a consequence of our approach is getting NAM regularization. The noticeable structures in conjunction with sharpened facets point out the advantage of our technique of NAM. In this research, we measure the performance of the clogging filter imaging protocol (auto-focus approach), and that is recommended in this 
particular paper. We analyzed the image taken by Neuroendoscopy unit and patient was treated at Multi Speciality Hospital, Pune, India.

\section{DISCUSSION}

Despite the fact that Tumor growths are much less widespread as compared to tumor polyploidy wounds, ripped skin lesions possess higher cancerous prospective. Ripped wounds are likely, even so, to be more challenging to diagnose employing a conventional technique in comparison with polyploidy growths, particularly for less-experienced neuro endoscopists. Our outcomes discovered that NAM offered excellent visibility as compared to that of SVM/PSO method. AI provided high-quality visual images of sideways scattering growths non-granular type. Some other scientific studies have claimed that SVM is relatively a good choice for differential medical diagnosis. Even so, regardless of whether PSO is helpful for tumor cancerous growth prognosis continues to be questionable, with experts confirming combined results [9].

Also, SVM Neuroendoscopy having a transparent cover discovered much more tumor neoplasias as compared to performed PSO with no transparent cover, even though carried out using less-experienced doctors [20]. The modern era of PSO has been consequently formulated along with the objective of enhanced perfection, despite the fact that possible tests analyzing this declare haven't been recently released. In our analysis, there seemed to be a significant difference in the standard of visual images of ripped cancers among NAM and SVM/PSO. Our results applying NAM which has a new technique found ensuring; nonetheless, there was clearly no diminished field of vision utilizing NAM in comparison with PSO. This particular outcome pointed out some likelihood of NAM pre-Neuroendoscopy ground search. Additional research is necessary to uncover the practical use of the field of vision along with delectability of ripped wounds while using the new era of NAM approach. Our outcomes demonstrated that NAM technique could be better than PSO Neuroendoscopy images for the recognition of ripped tumors region visibility.

\section{CONCLUSION}

In summary, our data revealed that NAM is a good choice for enhancing image quality throughout cases of tumor ripped cancers. In this particular paper, we've advised an NAM semi-local clogging - a layeraliasing method using NAM algorithm to obtain good quality images which can be utilized for pre-surgical and post-surgical initial investigations. This technique can not only achieve the excellent quality of images but also lessen aliasing artifacts of images significantly so that doctors can visualize tumor region very well. As per our implementation results and physician's opinion, NAM can be very useful as an offline tool. Also concerning upcoming work, it is appropriate undertakes a technique of effectively fortifying the computational performance within our algorithm so as to entail considerably larger research region associated with anti-aliasing. And proper software package API may be designed to apply for real time Neuroendoscopy system development.

\section{ACKNOWLEDGEMENTS}

We are thankful to the Department of Computer Science \& Engineering, K. L. University, A. P. India for providing the research fascilities., Vishwakarma Institute of Technology, Pune and Sinhgad Academy of Engineering, Pune for providing the support and guidance for this Research work. We are thankful to Dr. M. R. Narasinga Rao, Dr. D. Rajeswara Rao, and Dr. N. Srinivas from K. L. University, A. P., India for their motivation, guidance and support for this work. We are also thankful to Dr. K. P. Patil, Principal and Prof. A. N. Adapanawar, HOD-IT from Sinhgad Academy of Engineering, Pune. We are thankful to all the Members of the Hospital.

\section{REFERENCES}

[1] Ryalat, Mohammad Hashem, et al., "Evaluation of Particle Swarm Optimisation for Medical Image Segmentation", International Conference on Systems Science 2016. Springer International Publishing, vol. 539, pp. 61-72, Nov 2016.

[2] Chen, Ruey-Maw, and Chuin-Mu Wang, "MRI Brain Tissue Classification Using Unsupervised Optimized Extenics-Based Methods", 2016 International Symposium on Computer, Consumer and Control (IS3C), IEEE, July 2016.

[3] Mehdi Hosseini, et al., "Estimation of soil mechanical resistance parameter by using particle swarm optimization, genetic algorithm and multiple regression methods", Soil and Tillage Research, vol. 157, pp. 32-42, 2016.

[4] Zhao, Cuijie, and Guozhen Wang. "Application of Chaotic Particle Swarm Optimization in Wavelet Neural Network." TELKOMNIKA (Telecommunication Computing Electronics and Control), 12.4, (2014): 997-1004. 
[5] Nichat, Aparna M., and S. A. Ladhake, "Brain Tumor Segmentation and Classification Using Modified FCM and SVM Classifier.", IJARCCE, Vol. 5, Issue. 4, 2016.

[6] Yudistira, Novanto, and Daut Daman. "Issues and Problems in Brain Magnetic Resonance Imaging: An Overview." TELKOMNIKA, 6.1 (2008): 57-64.

[7] Abbasi, Solmaz, and Farshad Tajeripour, "Detection of brain tumor in 3D MRI images using local binary patterns and histogram orientation gradient.", Neurocomputing, 219 (2017): 526-535.

[8] R. Trigui, et al., "Automatic classification and localization of prostate cancer using multi-parametric MRI/MRS", Biomedical Signal Processing and Control, vol. 31, pp 189 - 198, 2017.

[9] Pizarro, Ricardo A., et al. "Automated Quality Assessment of Structural Magnetic Resonance Brain Images Based on a Supervised Machine Learning Algorithm", Frontiers in Neuroinformatics, 10 (2016).

[10] Jothi, G. "Hybrid Tolerance Rough Set-Firefly based supervised feature selection for MRI brain tumor image classification.", Applied Soft Computing, vol.45.C, pp.639-651, (2016).

[11] Hu, Dewen, "Brain Pattern Analysis Based on Magnetic Resonance Imaging", Advances in Cognitive Neurodynamics (V), Springer Singapore, 2016, pp. 49-51.

[12] S. L. Bangare, et. al., "Implementing Tumor Detection and Area Calculation in MRI Image of Human Brain Using Image Processing Techniques", Int. Journal of Engineering Research and Applications, vol.5, I.4, Part 6, (2015), pp.60-65..

[13] Snehkunj, Rupal, et. al., "Pattern Detection Framework for MRI Images and Labeling Volume of Interest (VoI)", Proceedings of the Second International Conference on Computer and Communication Technologies. Springer India, 2016.

[14] Pieciak, Tomasz, et. al., "Non-Stationary Rician Noise Estimation in Parallel MRI using a Single Image: a Variance-Stabilizing Approach", IEEE Transactions on Pattern Analysis and Machine Intelligence, (2016).

[15] Picot, Fabien, et. al. "Imaging system based on diffusive reflectance spectroscopy for blood vessels detection during brain biopsy procedure." Photonics North (PN), 2016. IEEE, 2016.

[16] Mohyeldin, Ahmed, Russell R. Lonser, and J. Bradley Elder. "Real-time magnetic resonance imaging-guided frameless stereotactic brain biopsy: technical note", Journal of neurosurgery, 124.4 (2016): 1039-1046.

[17] Le Nobin, Julien, et. al. "Image guided focal therapy for magnetic resonance imaging visible prostate cancer: defining a 3-dimensional treatment margin based on magnetic resonance imaging histology co-registration analysis", The Journal of Urology, 194.2 (2015): 364-370.

[18] Kang, Byungjeon, et. al. "Towards accurate robot-assisted neuroendoscopy using an ergonomic handling interface and a lightweight robot", 2014 36th Annual International Conference of the IEEE Engineering in Medicine and Biology Society. IEEE, (2014): 6876-6879.

[19] Çetingül, H. Ertan, et. al., "Segmentation of high angular resolution diffusion MRI using sparse Riemannian manifold clustering", IEEE transactions on Medical Imaging, 33.2 (2014): 301-317.

[20] Sachdeva, Jainy, et al., "A package-SFERCB Segmentation, feature extraction, reduction and classification analysis by both SVM and ANN for brain tumors", Applied Soft Computing, vol. 47 (2016), pp.151-167.

[21] Zhang, Yudong, et. al., "An MR brain images classifier system via particle swarm optimization and kernel support vector machine", The Scientific World Journal, Vol. 2013 (2013).

[22] Zhang, Yu-Dong, et al. "Magnetic resonance brain image classification based on weighted-type fractional Fourier transform and nonparallel support vector machine", International Journal of Imaging Systems and Technology, 25.4 (2015): 317-327.

[23] Fachrurrozi, Muhammad, and Muhammad Mujtahid, "Iris Image Recognition Based on Independent Component Analysis and Support Vector Machine", TELKOMNIKA (Telecommunication Computing Electronics and Control) 13.2 (2015): 597-603.

[24] Zhou, Xing-Xing, et. al., "Combination of stationary wavelet transform and kernel support vector machines for pathological brain detection", Simulation (2016): 0037549716629227.

[25] Vasanth, P.C., and K.R. Nataraj. "Facial Expression Recognition Using SVM Classifier", Indonesian Journal of Electrical Engineering and Informatics (IJEEI) 3.1 (2015): 16-20.

[26] Alfonse, Marco, and Abdel-Badeeh M. Salem, "An Automatic Classification of Brain Tumors through MRI Using Support Vector Machine", Egyptian Computer Science Journal (ISSN: 1110-2586) 40.03 (2016).

[27] Lahmiri, Salim, and Mounir Boukadoum, "An evaluation of particle swarm optimization techniques in segmentation of biomedical images", Proceedings of the Companion Publication of the 2014 Annual Conference on Genetic and Evolutionary Computation. ACM, 2014., pp.1313-1320.

[28] Zhang, Yudong, Shuihua Wang, and Genlin Ji, "A comprehensive survey on particle swarm optimization algorithm and its applications", Mathematical Problems in Engineering 2015 (2015).

[29] Menashe Zaaroor, Gideon, et. al. "Real-time change detection of steady-state evoked potentials", Biological cybernetics 107.1 (2013): 49-59.

[30] Couceiro, Micael S., et. al,. "Introducing the fractional-order Darwinian PSO", Signal, Image and Video Processing 6.3 (2012): 343-350.

[31] Maitra, Madhubanti, and Amitava Chatterjee., "A hybrid cooperative-comprehensive learning based PSO algorithm for image segmentation using multilevel thresholding", Expert Systems with Applications 34.2 (2008): 1341-1350.

[32] Sutikno, Tole, Mochammad Facta, and GR Arab Markadeh. "Progress in Artificial Intelligence Techniques: from Brain to Emotion", TELKOMNIKA (Telecommunication Computing Electronics and Control) 9.2 (2011): 201-202.

[33] Abdar, Moloud, et al., "Comparing Performance of Data Mining Algorithms in Prediction Heart Diseases", International Journal of Electrical and Computer Engineering (IJECE) 5.6 (2015): 1569-1576. 
[34] S. L. Bangare, G. Pradeepini and S. T. Patil, "Brain Tumor Classification Using Mixed Method Approach", IEEE International Conference on Information Communication and Embedded Systems (ICICES), S.A.Engineering College, Chennai, Tamilnadu, India, (2017).

[35] S. L. Bangare, G. Pradeepini et al., "Brain Tumor Classification Using Support Vector Machine: A Review", National Conference on Computational Sciences (NCCS), Karpagam University, India, (2017).

[36] S. L. Bangare, et al. "Reviewing Otsu's Method for Image Thresholding", International Journal of Applied Engineering Research 10.9 (2015): 21777-21783.

\section{BIOGRAPHIES OF AUTHORS}

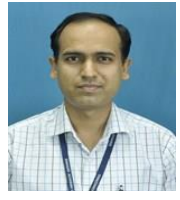

Mr Sunil L. Bangare is Ph.D. Research Scholar at Department of CSE, K. L. University, Vaddeswaram, Andhra Pradesh, India and working as Assistant Professor, Sinhgad Academy of Engineering, Pune, India. Published papers in International Journals, National and International Conferences. Contributed few chapters in 04 books. He is having more than 10 years of teaching and industry experience and Main research interest includes Image Processing, Biomedical Engineering, Software Engineering and Machine Learning.

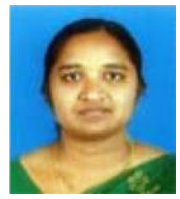

Dr. G Pradeepini is working as Professor, Department of Department of CSE, K. L. University, Vaddeswaram, Andhra Pradesh, India. Published papers in National \& International Journals and Conferences. She having 14 years of teaching experience and 7 years of research experience. Main research interest includes Data Mining and Data Warehouse, Big data and Neural Networks, Image Processing.

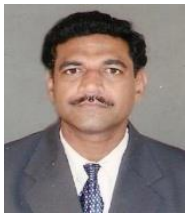

Dr. Shrishailappa T. Patil is working as Professor, Department of Computer Engineering, Vishwakarma Institute of Technology, Pune, India. Published papers in National \& International Journals and Conferences. He is having 28 years of teaching experience. Main research interest includes Digital Signal Processing, Image Processing, Neural Networks, Computer Networks, Mobile Computing, Microprocessor \& Microcontrollers, Embedded Systems, Biomedical Engineering. Bioinformatics. 